\title{
Letter
}

\section{Diamagnetic drift instabilities in collisional non-uniform quantum dusty magnetoplasmas}

\author{
M. J A M I L ${ }^{1}$, C H. U Z M A ${ }^{1}$, K. Z U B I A ${ }^{1}$, I. Z E B A ${ }^{1}$, H. M. R A F I Q U E \\ and M. S A L I M U L L A H ${ }^{3}$ \\ ${ }^{1}$ Department of Physics, GC University, Lahore-54000, Pakistan \\ (jamil.gcu@gmail.com) \\ ${ }^{2}$ Department of Physics, University of the Punjab, Lahore-54000, Pakistan \\ ${ }^{3}$ Department of Physics, Jahangirnagar University, Savar, Dhaka-1342, Bangladesh
}

(Received 17 December 2011; revised 13 March 2012; accepted 20 March 2012; first published online 17 April 2012)

\begin{abstract}
Dust-acoustic and dust-lower-hybrid diamagnetic drift wave instabilities have been examined in a collisional non-uniform quantum dusty magnetoplasma. The dust-acoustic drift instability arises through the Fermi degenerate pressure of electrons for a high-density plasma, while for a relatively low-density collisional quantum plasma and short wavelength consideration, the instability is dominated by the Bohm potential effect exciting a new quantum dust-acoustic wave. In the longrange wavelength limit, dust-lower-hybrid waves are found to be unstable because of the diamagnetic drift of magnetized ions. Various possible instability conditions are found for diamagnetic drift instability.
\end{abstract}

During the last two decades, low-frequency waves and instabilities in dusty plasmas with or without the presence of external magnetic fields have occupied the major areas of dusty plasma physics. This is because all properties of dusty plasmas in general are related with waves and instabilities. With the advent of dustacoustic (DA) and dust-lower-hybrid (DLH) waves (Rao et al. 1990; Salimullah et al. 1992; Shukla 1992; Shukla and Silin 1992; Salimullah 1996; Jamil et al. 2010) involving dust dynamics, a considerable amount of progress has been achieved in the context of astrophysical and laboratory dusty plasma systems. Earlier, cross-field instabilities were investigated in the presence of external electric and magnetic fields in possible laboratory dusty plasma experiments (Shukla et al. 2002; Rastunkov and Krainov 2004; Rosenberg and Shukla 2004; Norreys et al. 2009; Sabeen et al. 2010). In recent years, there has been a growing interest in quantum dusty plasmas because of their importance in micro-electronics and electronic devices with nano-electronic components, dense astrophysical objects, and in laser-produced plasmas (Kremp et al. 1999; Andreev 2000; Jung 2001; Opher et al. 2001; Chabrier et al. 2002; Haas et al. 2003; Bingham et al. 2004; Marklund and Shukla 2006; Shukla et al. 2006a; Brodin and Marklund 2007; Marklund and Brodin 2007; Brodin et al. 2008a; Ren et al. 2009; Salimullah et al. 2009a; Hussain et al. 2010). When a plasma is cooled to an extremely low temperature, the de Broglie wavelengths of plasma particles could be at least comparable to the scale lengths, such as the Debye length or the Larmor radius etc., in the system. In such plasmas, the ultra-cold dense plasma would behave as a Fermi gas, and quantum mechanical effects might play a vital role in the behavior of charge carriers of these plasmas under extreme conditions. There are different models for quantum plasmas. Here we are using the Schrodinger-Poisson model, which is a simplified model and capture the main features of quantum plasmas. It is more appropriate for the analytical and numerical treatment of problems. It makes direct use of global properties of plasma quantities, such as density and average velocity, and therefore may ignore the spin effects (Haas 2005; Haas et al. 2000; Asenjo et al. 2011).

Spin effects of quantized charges are important for linear modes in the presence of a strong magnetic field, and for the systems whose confined geometry is frequently comparable with the thermal de Broglie wavelengths like micro electronics, nano-wires, quantum dots, etc. In large dimensional systems, because of random orientation of spins, high-density collisional plasmas, and the systems where the electrostatic forces are strong as compared with spin forces, the macroscopic spin population may not sustain and therefore become negligible (Garcia et al. 2005; Shukla 2006; Shukla and Eliasson 2006; Brodin et al. 2008b).

Collision between the quantum mechanical identical particles is considerably different from those in classical plasmas because of their collision cross sections. An increase in plasma oscillation frequency in quantum plasmas suppresses the collision probability, e.g., electronelectron collision is negligibly small [ $\mathrm{Na}$ and Jung 2008]. 
In this paper, we study the diamagnetic drift instabilities of DA and DLH waves in an inhomogeneous and collisional quantum dusty magnetoplasma by including the collision frequency of ions and dust particles. We consider an infinitely extended inhomogeneous highdensity dusty magnetoplasma containing electrons, ions, and charged dust grains in the presence of a homogeneous static ambient magnetic field $\mathbf{B}_{0} \| \hat{\mathbf{z}}$. At equilibrium, we assume that the charge quasi-neutrality condition is satisfied, i.e., $n_{i 0}+\left(q_{d} / e\right) n_{d 0}=n_{e 0}$, where $n_{j 0}(x)$ is the equilibrium number density of the $j$ th species $(j=$ electrons, ions, or dust), $q_{d}$ is the average charge on a dust grain, and $e$ is the electronic charge. We analyze the stability properties of the system against electrostatic perturbations, including the effects of heavier species.

The governing equations in the quantum hydrodynamic (QHD) model (Gardner 1994, Gasser et al. 2000, Manfredi and Haas 2001; Haas 2005; Manfredi 2005; Ali and Shukla 2006; Shukla et al. 2006; Ren et al. 2008, Saleem et al. 2008) for electrons, ions, and charged dust grains $(j=e, i, d)$ in the presence of the ambient magnetic field $\mathbf{B}_{0}$ are

$$
\begin{aligned}
m_{j} n_{j 0} \frac{\partial}{\partial t} \mathbf{v}_{j}= & -n_{j 0} q_{j} \nabla \phi+n_{j 0} \frac{q_{j}}{c} \mathbf{v}_{j} \times \mathbf{B}_{0}-\nabla p_{F j 1} \\
& +\frac{\hbar^{2}}{4 m_{j}} \nabla\left(\nabla^{2} n_{j 1}\right) .
\end{aligned}
$$

The corresponding components of velocities for $j$ th species can be written from (1) as

$$
\mathbf{v}_{j}^{L}=\frac{q_{j} \Phi}{m_{j} \omega}\left[\frac{i \omega \omega_{c j}}{\omega^{2}-\omega_{c j}^{2}} k_{y} \hat{x}+\frac{\omega^{2}}{\omega^{2}-\omega_{c j}^{2}} k_{y} \hat{y}+k_{z} \hat{z}\right],
$$

where

and

$$
\Phi=\phi+\frac{m_{j}}{q_{j}} V_{F j}^{\prime 2} \frac{n_{j 1}}{n_{j o}}
$$

$$
V_{F j}^{\prime}=V_{F j}\left(1+\gamma_{j}\right)^{1 / 2}, \quad \gamma_{j}=\hbar^{2} k^{2} / 8 m_{e} K_{B} T_{F j},
$$

where $n_{j}=n_{j 0}+n_{j 1}, \hbar$ is the Planck's constant divided by $2 \pi, \phi(\mathbf{r}, t)$ is the electrostatic potential in the quantum magnetoplasma, and $q_{j}, m_{j}, n_{j}$, and $c$ are the charge, mass, total equilibrium number density with equilibrium value $n_{j 0}$ of the $j$ th species, and the velocity of light in vacuum, respectively. Here we take into account the quantum effects of all the species in general when they are considered extremely cold. In (1), we assume that the plasma particles in a zero-temperature Fermi gas satisfy the pressure law (Manfredi and Haas 2001; Manfredi 2005), $p_{F j}=m_{j} V_{F j}^{2} n_{j}^{3} / 3 n_{j 0}^{2}$, where $V_{F j}=\left(2 K_{B} T_{F j} / m_{j}\right)^{1 / 2}$ is the Fermi speed; $K_{B}, T_{F j}$, and $n_{j}$ are the Boltzmann constant, the Fermi temperature, and the total number density with its equilibrium value $n_{j 0}$, respectively.

It may be mentioned that in a three-dimensional quantum plasma, the total pressure should be proportional to $n^{(N+2) / N}$, where $N=3$. However, according to Manfredi and Haas (2001) this choice is not good, as the results of QHD model differ from the Wigner-
Poisson model. Therefore, the total pressure of the threedimensional quantum plasma is approximately described by the one-dimensional Fermi pressure. Similar pressure law was employed by Ali and Shukla (2006) for threedimensional quantum plasmas.

The equation of continuity for inhomogeneity along $x$-direction is

$$
\frac{\partial n_{j}}{\partial t}+\nabla \cdot\left(n_{j} \mathbf{v}_{j}\right)=0
$$

After linearization of continuity equation, we have

$$
\frac{\partial n_{j 1}}{\partial t}+n_{j 0}\left(\nabla . \mathbf{v}_{j y}+\nabla . \mathbf{v}_{j z}\right)+\mathbf{v}_{j x} . \partial n_{j 0} / \partial x=0 .
$$

The Fourier transformation of (4) gives the following equation:

$$
n_{j 1}^{L}=\frac{n_{j 0}}{\omega} k_{z} v_{j z}^{L}+\frac{n_{j 0}}{\omega} k_{y} v_{j y}^{L}+\frac{n_{j 0}^{\prime}}{i \omega} v_{j x}^{L} .
$$

Further, the Poisson's equation satisfying the electrostatic potential $\phi$ of electrostatic perturbation is

$$
\nabla^{2} \phi=4 \pi e\left(n_{e 1}-n_{i 1}-\frac{q_{d}}{e} n_{d 1}\right)
$$

In the presence of density inhomogeneities in $x$ direction and the ambient magnetic field, $\mathbf{B}_{0}=\hat{\mathbf{z}} B_{0}$, we assume the presence of drift waves propagating in the $y z$-plane, proportional to $\exp \left[-i\left(\omega t-k_{y} y-k_{z} z\right)\right]$, where $k^{2}=k_{y}^{2}+k_{z}^{2}$ and $k_{y}^{2} \gg k_{z}^{2}$. Here $\omega$ and $\mathbf{k}$ are angular frequency and wavenumber vector, respectively.

We would like to mention that for weak inhomogeneity approximation (Ren et al. 2008) with $k_{x} \ll k$ and $k \gg 1 / L_{j}$, we can retain the dominant linear term in the Bohm potential term of the equation of motion. Similar consideration has been employed earlier in the literature (Shukla et al. 2006b) for drift wave investigations in the non-uniform quantum magnetoplasmas. However, the non-uniformity of equilibrium density is taken through the continuity equation to study drift waves in nonuniform quantum plasmas.

Using velocity components from (2) into (5), we have

$$
n_{j 1}^{L}=\frac{n_{j 0} q_{j}}{\omega}\left[\frac{k_{z}^{2}}{\omega^{2}}+\frac{k_{y}^{2}}{\omega^{2}-\omega_{c j}^{2}}\left(1-\frac{\omega_{c j}}{k_{y} L_{j} \omega}\right)\right] \Phi .
$$

After putting $\Phi$

$$
n_{j 1}^{L}=\frac{\frac{n_{j 0} q_{j}}{\omega}\left[\frac{k_{z}^{2}}{\omega^{2}}+\frac{k_{y}^{2}}{\omega^{2}-\omega_{c j}^{2}}\left(1-\frac{\omega_{c j}}{k_{y} L_{j} \omega}\right)\right] \phi}{1-V_{F j}^{\prime 2}\left[\frac{k_{z}^{2}}{\omega^{2}}+\frac{k_{y}^{2}}{\omega^{2}-\omega_{c j}^{2}}\left(1-\frac{\omega_{c j}}{k_{y} L_{j} \omega}\right)\right]} .
$$

We know,

$$
n_{j 1}^{L}=-\frac{1}{4 \pi q_{j}} \chi_{j} k^{2} \phi
$$

From (8) and (9), we obtain the dielectric susceptibility for $j$ th species, where $j=e, i, d$ as (Baines et al. 1965; Salimullah et al. 2009b)

$$
\chi_{j}=-\frac{\omega_{p j}^{2}\left[\frac{k_{z}^{2}}{\omega^{2}}+\frac{k_{y}^{2}}{\omega^{2}-\omega_{c j}^{2}}\left(1-\frac{\omega_{c j}}{k_{y} L_{j} \omega}\right)\right]}{k^{2}-k^{2} V_{F j}^{\prime 2}\left[\frac{k_{z}^{2}}{\omega^{2}}+\frac{k_{y}^{2}}{\omega^{2}-\omega_{c j}^{2}}\left(1-\frac{\omega_{c j}}{k_{y} L_{j} \omega}\right)\right]},
$$


where $\omega_{p j}=\left(4 \pi n_{j 0} q_{j}^{2} / m_{j}\right)^{1 / 2}$ and $\omega_{c j}=q_{j} B_{0} / m_{j} c$ are the plasma frequency and the cyclotron frequency of the $j$ th species, respectively. In (5), the scale length of inhomogeneity, $L_{j}=-n_{j 0} / n_{j 0}^{\prime}$ with $n_{j 0}^{\prime}=\partial n_{j 0}(x) / \partial x$. For quantum effect involving electron dynamics only, one can write $k^{2} \lambda_{F e}^{\prime 2}=k^{2} \lambda_{F e}^{2}+\omega_{Q e}^{2} / 4 \omega_{p e}^{2}$, where $\lambda_{F e}=V_{F e} / \omega_{p e}$ and $\omega_{Q e}=\hbar k^{2} / m_{e}$. We use (10) to find the general dielectric response function $\epsilon(\omega, \mathbf{k})$ of non-uniform quantum dusty magnetized plasma under various possible conditions,

$$
\epsilon(\omega, \mathbf{k})=1+\chi_{e}(\omega, \mathbf{k})+\chi_{i}(\omega, \mathbf{k})+\chi_{d}(\omega, \mathbf{k}) .
$$

It may be mentioned here that in the presence of considerable amount of neutral atoms/molecules in the dusty plasma, collision frequencies of plasma species with neutrals cannot be ignored. Also, the diamagnetic drift velocities of magnetized species in the non-uniform plasma may be important that might cause diamagnetic drift wave instabilities. In the presence of the ion diamagnetic drift velocity, $\mathbf{u}_{D i}=\left(c T_{F i} / n_{i 0} e B_{0}\right)\left(\partial n_{i 0}(x) / \partial x\right) \hat{\mathbf{y}}$, and the average collision frequency, $v_{i}$, the $\omega$ in (11) should be properly taken into account by replacing $\omega$ with $\omega-k_{y} u_{D i}+i v_{i}$. We ignore diamagnetic drifts of electrons and dust grains because they are not assumed magnetized.

In order to study the diamagnetic DA and DLH drift wave instabilities in a collisional non-uniform quantum dusty plasma in the presence of an external static and homogeneous magnetic field, we assume

$$
\begin{aligned}
\omega & \leqslant \omega_{i}^{*} \ll \omega_{c i}, \quad k_{z}^{2} \ll k_{y}^{2}, \\
k V_{F i}^{\prime} & \ll \omega \ll k V_{F e}^{\prime},
\end{aligned}
$$

where $\omega_{i}^{*}=\omega_{p i}^{2} / k_{y} L_{i} \omega_{c i}$ is the drift frequency of cold and magnetized ions.

Dust-acoustic and DLH drift wave instabilities are presented in the following sections.

\section{Dust-acoustic drift wave instabilities $\left(1 / k^{2} \lambda_{F e}^{\prime 2} \simeq 1 / k^{2} \lambda_{F e}^{2} \gg f_{i} \gg 1\right)$}

First, we consider a high-density quantum dusty plasma where quantum effect arises through the Fermi degenerate pressure of electrons and the small Bohm potential is neglected. Here we consider that the electrons fully degenerate with the Fermi temperature $T_{F e}$, ions are magnetized and collisional, and the dust grains are unmagnetized but collisional (Baines et al. 1965; Rosenberg and Shukla 2004). In this case, the dielectric function satisfying (12) is given by

$$
\epsilon(\omega, \mathbf{k})=1+\frac{1}{k^{2} \lambda_{F e}^{2}}+f_{i}-\frac{\omega_{i}^{*}}{\Omega_{i}+i v_{i}}-\frac{\omega_{p d}^{2}}{\omega\left(\omega+i v_{d}\right)},
$$

where $\Omega_{i}=\omega-k_{y} u_{D i}$ and $f_{i}=\omega_{p i}^{2} / \omega_{c i}^{2}$; here $u_{D i}$ is the magnetized ion fluid drift velocity. For $i v_{i} \gg \Omega_{i}, i v_{d} \ll \omega$, and $1 / k^{2} \lambda_{F e}^{2} \gg f_{i}$, the above equation for $\epsilon=0$ can be written as

$$
1+\frac{i \omega_{p i}^{2}}{k_{y} L_{i} \omega_{c i}} \frac{k^{2} \lambda_{F e}^{2}}{v_{i}}-\frac{k^{2} C_{F d}^{2}}{\omega^{2}}\left(1-\frac{i v_{d}}{\omega}\right)=0
$$

where $C_{F d}^{2}=\omega_{p d}^{2} V_{F e}^{2} / \omega_{p e}^{2}$. The relation $\epsilon_{r}=0$, where $\epsilon_{r}$ is the real part of the dielectric function, yields the dispersion relation of the modified DA wave in the Fermi quantum dusty plasma

$$
\omega_{D A}=k C_{F d}
$$

Assuming $k_{y} L_{i} \omega_{c i}<k C_{F i}$, where $C_{F i}=\omega_{p i} \lambda_{F e}$ is the ionacoustic speed at the electron-Fermi temperature, (14) describes the DA drift instability given by

$$
\frac{\omega}{\omega_{D A}}=\frac{1+i}{\sqrt{2}}\left(\frac{v_{i}\left|L_{i}\right| \omega_{c i}}{k_{y} C_{F i}^{2}}\right)^{1 / 2}-\frac{i v_{d}}{2 \omega_{D A}},
$$

where $L_{i}=-\left|L_{i}\right|$ for a positive ion density gradient. We note that DA wave in the Fermi quantum dusty plasma grows in amplitude when the ion inhomogeneity scale length is sufficiently small.

Next, we consider the strong quantum effect arising through the Bohm potential compared with the effect arising through the Fermi degenerate pressure. This situation arises for a relatively low-density quantum plasma and short wavelength DA waves. In this case, the dispersion relation is obtained from

$$
1+\frac{4 \omega_{p e}^{2}}{\omega_{Q e}^{2}}+f_{i}-\frac{\omega_{i}^{*}}{\Omega_{i}}\left(1-\frac{i v_{i}}{\Omega_{i}}\right)-\frac{\omega_{p d}^{2}}{\omega^{2}}\left(1-\frac{i v_{d}}{\omega}\right)=0,
$$

where $\Omega_{i}>i v_{i}$ is made.

However, for $i v_{i} \gg \Omega_{i}$ and $i v_{d} \ll \omega,(14)$ reduces to

$$
1-i x-\frac{\omega_{p d}^{2} /\left(4 \omega_{p e}^{2} / \omega_{Q e}^{2}\right)}{\omega^{2}}\left(1-\frac{i v_{d}}{\omega}\right)=0,
$$

where

$$
x=\frac{\delta \hbar^{2} k^{4}}{4 m_{e} m_{i} v_{i}} \frac{1}{k_{y}\left|L_{i}\right| \omega_{c i}},
$$

with non-neutrality parameter in the dusty plasma, $\delta=$ $n_{i 0} / n_{e 0}$. Assuming $\delta \hbar^{2} k^{4} \gg 4 m_{e} m_{i} k_{y}\left|L_{i}\right| \omega_{c i} v_{i}$, the DA drift instability is described by

$$
\frac{\omega}{\omega_{Q D A}}=\frac{1+i}{\sqrt{2}}\left(\frac{4 m_{e} m_{i} v_{i} k_{y}\left|L_{i}\right| \omega_{c i}}{\delta \hbar^{2} k^{4}}\right)^{1 / 2}-\frac{i v_{d}}{2 \omega_{Q D A}}
$$

where the quantum DA wave frequency because of the Bohm potential is given by

$$
\begin{aligned}
\omega_{Q D A} & =\frac{\omega_{p d} \omega_{Q e}}{2 \omega_{p e}} \\
& =\frac{\hbar k^{2} Z_{d}}{2 \sqrt{m_{e} m_{d}}} \sqrt{\frac{n_{d 0}}{n_{e 0}}} .
\end{aligned}
$$

Here we note that the quantum modified DA wave suffers instability for small $v_{i}\left|L_{i}\right|$. 


\section{Dust-lower-hybrid drift wave instabilities}

$$
\left(f_{i} \gg 1 / k^{2} \lambda_{F e}^{2} \gg 1\right)
$$

For $i v_{i} \gg \Omega_{i}$ and $i v_{d} \ll \omega$ in this case, the dielectric function from (13) reduces to

$$
\epsilon(\omega, \mathbf{k})=1+i\left(\frac{\omega_{p i}^{2}}{k_{y} L_{i} v_{i} \omega_{c i} f_{i}}\right)-\frac{\omega_{d l h}^{2}}{\omega^{2}}\left(1-\frac{i v_{d}}{\omega}\right),
$$

where the DLH frequency is given by $\omega_{d l h}=\omega_{p d} \omega_{c i} / \omega_{p i}$. For a positive density gradient, $L_{i}=-\left|L_{i}\right|$, the dispersion relation for a growing DLH drift wave is obtained from (22) as,

$$
1-\frac{i \omega_{c i}}{v_{i} k_{y}\left|L_{i}\right|}-\frac{\omega_{d l h}^{2}}{\omega^{2}}\left(1-\frac{i v_{d}}{\omega}\right)=0 .
$$

Thus, the DLH instability for $\omega_{c i} \gg v_{i} k_{y}\left|L_{i}\right|$ is given by

$$
\frac{\omega}{\omega_{d l h}}=\frac{1+i}{\sqrt{2}}\left(\frac{v_{i} k_{y}\left|L_{i}\right|}{\omega_{c i}}\right)^{1 / 2}-\frac{i v_{d}}{2 \omega_{d l h}} .
$$

Obviously, the long-wavelength DLH wave possesses instability in the inhomogeneous dusty plasma.

Next, we consider $i v_{i} \ll \Omega_{i}$ and $i v_{d} \ll \omega$. Thus, from (13) we have

$$
1+\frac{\omega_{c i}}{k_{y}\left|L_{i}\right|\left(\omega-k_{y} u_{D i}\right)}-\frac{\omega_{d l h}^{2}}{\omega^{2}}=0
$$

For $k_{y} u_{D i}>\omega$, we obtain a purely growing DLH instability from

$$
\omega^{2}=-\frac{\omega_{d l h}^{2} k_{y}^{2}\left|L_{i}\right| u_{D i}}{\omega_{c i}} .
$$

The growth rate of the purely growing DLH instability depends on the diamagnetic drift velocity of ions.

In summary, we have investigated the DA and DLH diamagnetic drift wave instabilities in the presence of a static ambient or an applied magnetic field in a collisional non-uniform quantum dusty plasma. For the high-density plasma, the quantum effect on DA drift instability arises through the Fermi degenerate pressure. However, for a relatively low-density quantum plasma (e.g., nano-scale microelectronics, semiconductor plasmas, or laser-produced plasmas) and for short wavelength, the quantum effect is taken through the Bohm potential of quantum plasma. Various possible instability conditions are found for the diamagnetic DA wave instabilities. The linear dispersion relations of DA wave are also found in quantum plasma (cf. Opher et al. 2001; Shukla and Stenflo 2006a). In the long wavelength approximation, we find conditions for the DLH diamagnetic ion drift instabilities where the quantum effect is not significant for the inhomogeneous quantum dusty plasma.

We would like to stress here that in quantum dusty magnetoplasmas, such as dense astrophysical environments, microelectronics, nano-structured materials, high-density laser-produced plasmas, etc., the spatial non-uniformity of parameters, particularly the plasma densities of species, may be obviously quite common. Equilibrium density non-uniformity in the presence of magnetic fields gives rise to electrostatic drift waves in quantum systems. Various possible instability conditions are found for electrostatic diamagnetic drift instabilities. These instabilities would excite possible electrostatic drift waves, which give rise to the fundamental properties of non-uniform quantum plasmas. Electromagnetic drift waves must also be explored in quantum dusty magnetoplasmas which are beyond the scope of the present paper. The nonlinear wave-wave interactions at large-amplitude appear to be of interest also giving new properties of non-uniform dusty quantum magnetoplasmas. The parametric cascading of electromagnetic drift waves at large amplitudes in the presence of electrostatic drift waves may also lead to the turbulence of quantum plasmas, giving rise to various nonlinear effects.

\section{Acknowledgement}

One of the authors (M. Jamil) would like to thank M. Shahid for fruitful discussions. We would also like to thank anonymous referee(s) for constructive suggestions to improve this paper.

\section{References}

Ali, S. and Shukla, P. K. 2006 Potential distributions around a moving test charge in quantum plasmas. Phys. Plasmas 13, 102112.

Andreev, A. V. 2000 Self-consistent equations for the interaction of an atom with an electromagnetic field of arbitrary intensity. JETP Lett. 72, 238.

Asenjo, F. A., Muñoz, V., Vladivia J. A. and Mahajan, S. M. 2011 A hydrodynamical model for relativistic spin quantum plasmas. Phys. Plasmas 18, 012107.

Baines, M. F., Williams, I. P. and Asebiomo, A. S. 1965 Resistance to the motion of a small sphere moving through a gas. Mon. Not. R. Astron. Soc. 130, 63.

Bingham, R., Mendonca, J. T. and Shukla, P. K. 2004 Plasmabased charged-particle accelerators. Plasma Phys. Control. Fusion 46, R1.

Brodin, G. and Marklund, M. 2007 Spin solitons in magnetized pair plasmas. Phys. Plasmas 14, 112107.

Brodin, G., Marklund, M. and Manfredi, G. 2008a Quantum plasma effects in classical regime. Phys. Rev. lett. 100, 175001.

Brodin, G., Marklund, M., Zamanian, J., Ericsson, A. and Mana, P. L. 2008b Effects of the g factor in semiclassical kinetic plasma theory. Phys. Rev. Lett. 101, 245002.

Chabrier, G., Douchin, F. and Potekhin, A. Y. 2002 Dense astrophysical plasmas. J. Phys.: Condens. Matter 14, 9133.

Garcia, L. G., Haas, F., de Oliviera, L. P. L. and Goedert, J. 2005 Modified Zakharov equations for plasmas with a quantum correction. Phys. Plasmas 12, 012302.

Gardner, C. 1994 The quantum hydrodynamic model for semiconductor devices. SIAM J. Appl. Math. 54, 409.

Gasser, I., Lin, C. K. and Markowich, P. 2000 A review of dispersive limits of (non)linear Schrodinger-type equations. Taiwanese J. Math. 4, 501.

Hass, F. 2005 A magnetohydrodynamic model for quantum plasmas. Phys. Plasmas 12, 062117.

Haas, F., Garcia, L. G., Goedert, J. and Manfredi, G. 2003 Quantum ion-acoustic waves. Phys. Plasmas 10, 3858. 
Haas, F., Manfredi, G. and Feix, M. R. 2000 A multistream model for quantum plasmas. Phys. Rev. E 62, 2763.

Hussain, A., Zeba, I., Salimullah, M., Murtaza, G. and Jamil, M. 2010 Modified screening potential in a high-density inhomogeneous quantum dusty magnetoplasma. Phys. Plasmas 17, 054504.

Jamil, M., Shah, H. A., Salimullah, M., Zubia, K., Zeba, I. and Uzma, Ch. 2010 The parametric decay of Alfven waves into shear Alfvén waves and dust lower hybrid waves. Phys. Plasmas 17, 073703.

Jung, Y. D. 2001 Quantum-mechanical effects on electronelectron scattering in dense high-temperature plasmas. Phys. Plasmas 8, 3842.

Kremp, D., Bornath, Th., Bonitz, M. and Schlanges, M. 1999 Quantum kinetic equation for the interaction of dense plasmas with laser fields. Phys. Rev. E 60, 4725.

Manfredi, G. 2005 How to model quantum plasmas. Fields Inst. Commun. 46, 263.

Manfredi, G. and Haas, F. 2001 Self-consistent fluid model for a quantum electron gas. Phys. Rev. B 64, 075316.

Marklund, M. and Brodin, G. 2007 Dynamics of spin-1/2 quantum plasmas. Phys. Rev. Lett. 98, 025001.

Marklund M. and Shukla, P. K., 2006 Photonic crystal heterostructures and interfaces. Rev. Mod. Phys. 78, 455.

$\mathrm{Na}$, S. C. and Jung, Y. D. 2008 Oscillatory screening and quantum interference effects on electron collisions in quantum plasmas. Phys. Lett. A 372, 5605

Norreys, P. A., Beg, F. N., Sentoku, Y., Silva, L. O., Smith, R. A. and Trines, R. M. G. M. 2009 Intense laser-plasma interactions: new frontiers in high-energy density physics. Phys. Plasmas 16, 041002.

Opher, M., Silva, L. O., Dauger, D. E., Decyk, V. K. and Dawson, J. M. 2001 Nuclear reaction rates and energy in stellar plasmas: the effect of highly damped modes. Phys. Plasmas 8, 2454.

Rao, N. N., Shukla, P. K. and Yu, M. Y. 1990 Dust-acoustic waves in dusty plasmas. Planet. Space Sci. 38, 543.

Rastunkov V. S. and Krainov, V. P. 2004 Relativistic electron drift in over dense plasma produced by a super intense femtosecond laser pulse. Phys. Rev. E 69, 037402.

Ren, H., Wu, Z., Cao, J. and Chu, P. K. 2008 Electromagnetic drift waves in nonuniform quantum magnetized electronpositron-ion plasmas. J. Phys. A Math. Theory 41, 115501.
Ren, H., Wu, Z., Cao, J. and Chu, P. K. 2009 Electrostatic drift modes in quantum dusty plasmas with Jeans terms. Phys. Plasmas 16, 103705.

Rosenberg, M. and Shukla, P. K. 2004 Low-frequency drift instabilities in a strongly magnetized collisional dusty plasma. Plasmas Phys. Control. Fusion 46, 1807.

Sabeen T., Mirza, A. M. and Masood, W. 2010 Electrostatic drift-wave instability in a nonuniform quantum magnetoplasma with parallel velocity shear flows. Phys. Plasmas 17, 102705.

Saleem, H., Ali, A. and Khan, S. A. 2008 Low-frequency electrostatic and electromagnetic modes in nonuniform cold quantum plasmas. Phys. Plasmas 15, 014503.

Salimullah, M. 1996 Low-frequency dust-lower-hybrid modes in a dusty plasma. Phys. Lett. A 215, 296.

Salimullah, M., Hassan, M. H. A. and Sen, A. 1992 Lowfrequency electrostatic modes in a magnetized dusty plasma. Phys. Rev. A 45, 5929.

Salimullah, M., Jamil, M., Shah, H. A. and Murtaza, G. 2009a Jeans instability in a quantum dusty magnetoplasma. Phys. Plasmas 16, 014502.

Salimullah, M., Jamil, M., Zeba, I., Uzma, Ch. and Shah, H. A. 2009 b Drift wave instability in a nonuniform quantum dusty magnetoplasma. Phys. Plasmas 16, 034503.

Salimullah, M. and Sen, A. 1992 Low-frequency response of a dusty plasma. Phys. Lett. A 163, 82.

Shukla, P. K. 1992 Low-frequency modes in dusty plasmas. Phys. Scripta 45, 504.

Shukla, P. K. 2006 A new dust mode in quantum plasmas. Phys. Lett. A 352, 242.

Shukla, P. K. and Eliasson, B. 2006 Formation and dynamics of dark solitons and vortices in quantum electron plasmas. Phys. Rev. Lett. 96, 245001.

Shukla, P. K., Salimullah, M. and Sorasio, G. 2002 Some cross-field instabilities in magnetized dusty plasmas. Phys. Plasmas. 9, 5121.

Shukla, P. K. and Silin, V. P. 1992 Dust ion acoustic waves. Phys. Scripta 45, 508.

Shukla, P. K. and Stenflo, L. 2006a Jeans instabilities in quantum dusty plasmas. Phys. Lett. A 355, 378.

Shukla, P. K. and Stenflo, L. 2006b New drift modes in a nonuniform quantum magnetoplasma. Phys. Lett. A 357, 229. 